Alfarama Journal of Basic \& Applied Sciences

Faculty of Science Port Said University https://ajbas.journals.ekb.eg

ajbas@sci.psu.edu.eg

http://sci.psu.edu.eg/en/

January 2022, Volume 3 Issue I

DOI: $10.21608 / A J B A S .2021 .97158 .1068$

ISSN 2682-275X

Submitted: 20-09-2021

Accepted: 01-11-2021

Pages: 117-130

\title{
Pretopological applications for attribute reduction in information systems
}

\author{
Asmaa.M.Nasr ${ }^{1, *}$, Hewayda EIGhawalby ${ }^{1}$, R. Mareay $^{2}$ \\ ${ }^{1}$ Port Said University, Faculty of Engineering, Physics and Engineering Mathematics Department, Egypt. \\ ${ }^{2}$ KafrEl-sheikh University, Faculty of Science, Department of Mathematics, Egypt. \\ "Corresponding author: somanasr06@gmail.com
}

\begin{abstract}
In the past decades, the tremendous growth in computer-based information technology has led to the need for an efficient handling of information systems for the purpose of supporting decision making. The information systems are combinations of hardware and software that are used for storing and processing data; several techniques were introduced to extract useful information through the concepts of topological spaces. In this paper a pretopological space from an information system is to be constructed and examined, as well as defining some essential operations for the pretopological space and a brief study of its properties will be presented. Moreover, we are presenting a methodology for attributes reduction by gauging the accuracy degrees of decision sets in an information system.
\end{abstract}

\section{Keywords}

Pretopological Space; Closure Set; Interior Set; Information System.

\section{INTRODUCTION}

For the purpose of reducing the massive dimensionality of information mining of complicated structures; we propose a new method to reduce the attributes representing the information system under consideration, which may be considered as a pre-processing step. In literature there is a lot of research working on finding a way to reduce the attributes; among those are the rough set and the topological space concepts, beside other methods. However, there are some information system attributes that cannot be reduced using those methods.

In our proposed method, we use the concepts inherited from the pretopological spaces to construct a pretopology for the information system in hand, hence to use its properties to reduce the number of the its attributes.

Initially introduced by Marcel Brissaud, pretopology was established in 1975 as a branch of topology ([8],[15]). Despite naming Brissaud as the father of pretopology, for giving the first definition of a pretopological space [8], Belmandt was the one who developed those concepts and published the first book in 1993[7]; followed by a second book which was published in 2011[6]. And since, pretopology became an important tool that deals with mathematical models to measure the concepts of proximity and distance 
between sets and points; and started to play an important role in many different applications such as: economical modelling [14], data analysis [12], complex networks modelling ([12],[11]), modelling the impact of geographic proximity on scientific collaborations [5], data structure [13], and fuzziness and soft computing ([5],[9]).

The aim of this paper is to introduce a new methodology for measuring the degree of accuracy of information system on attributes. The remaining of this paper is structured as follows: In Section 2, the fundamental definitions from the pretopological space are introduced. In Section 3, developing a new type of a pretopological space used in information system by using dissimilarity method, And hence, an illustrative example showing the method of dissimilarity presented in Section 4. To conclude, Section 5, gives a brief conclusion.

\section{PRETOPOLOGICAL SPACE}

The concept of pretopology ([8],[15]) comes from the concept of topology, as the concept of topology can defined via open sets, neighborhoods and a Kuratowski closure operator ([10],[3],[4],[13]).

\subsection{Pretopological space}

For any set $X$, any given mapping $a: P(X) \rightarrow P(X)$ the pair $(X, a)$, is said to be a pretopological space, if it satisfies the following axioms:

i. $\quad a(\varnothing)=\emptyset$.

ii. $\quad A \subseteq a(A), \forall A \subseteq X$.

\subsection{Interior function}

For any set $(X, a)$, any interior function $i: P(X) \rightarrow P(X)$ defined as; $\operatorname{int}(A)=\operatorname{co}(a(\operatorname{co} A))$. where $\operatorname{co} A$ is complement of $A, A \subseteq X$.

\subsection{Information System}

An information system (IS, for short) [16] is an ordered triple $I S=(U, Q, V)$, such that $U$ is a nonempty finite set of objects (students, toy blocks, .....) called universe where $U=\left\{x_{1}, x_{2}, x_{3}, \ldots, x_{n}\right\}, Q$ is a nonempty set of attributes (colors, characteristic, .....) and $V$ is an attribute value set where $V=$ $\left\{v_{0}, v_{1}, v_{3}, \ldots, v_{n}\right\}$

\section{Measuring the Degree of Accuracy of Information System on Attributes (Dissimilarity Matrix)}

In our work, we aim to develop a new type of a pretopological space used in information system by using dissimilarity method.

\subsection{Definition}

Let $U=\left\{x_{1}, x_{2}, x_{3}, \ldots, x_{n}\right\}$ be a set of objects, and let $X \subseteq U$ be a set on $U$. Then,

$$
\zeta_{a}=x_{i} \cup\left\{x \in U / \mu\left(x_{i}, x_{j} \geq \delta\right), 1 \geq \delta \geq 0, i, j \in[1, n], n=|U|\right\}
$$

Where $\zeta_{a}$ is called a pretopological space used in information system.

Note: The cardinality of a set, which is basically the size of the set. The cardinality of a set is denoted by $|U|$.

\section{Lemma 3.1}

When choosing the values for $\mu$, the conditions of a pretopological space denoted in [6] must be considered.

\subsection{Definition}

For any set $\left(X, \zeta_{a}\right)$, any interior function $i: P(X) \rightarrow P(X)$ defined as;

$$
\operatorname{int}_{\zeta_{a}}(A)=\operatorname{co}\left(\zeta_{a}(\operatorname{co} A)\right) \text {. }
$$

where $\operatorname{co} A$ is complement of $A, A \subseteq X$. 


\subsection{Definition}

Given information system (IS) [17], defines a matrix $M_{A}$, called a dissimilarity matrix, each entry $M_{A}(x, y) \subseteq A$ consists of a set of attributes that can be discern between objects $x, y \in U$, and we can be described a matrix $M_{A}$ is an $|U| \times|U|$ matrix, and has the form:-

$$
M_{i j}(x, y)=\sum_{i=1}^{n} \sum_{j=1}^{n} \frac{\left(m_{x i}+m_{y i}\right)}{m_{x i} m_{y i}} \delta\left(x_{i}, y_{j}\right)
$$

where $\delta\left(x_{i}, x_{j}\right)=\left\{\begin{array}{lll}0 & \text { if } & \left(x_{i}=x_{j}\right) \\ 1 & \text { if } & \left(x_{i} \neq x_{j}\right)\end{array}\right.$

and $m_{x i}, m_{y i}$ are the numbers of objects that have the same categories $x_{i}$ and $y_{i}$ for attributes $i$.

where

$m_{x i} \rightarrow$ is the number of $m_{x i}$ iterations in his column.

$m_{y i} \rightarrow$ is the number of $m_{y i}$ iterations in his column.

\section{Lemma 3.3.1}

When constructing a dissimilarity matrix, we have to take into account that main diagonal elements are equal to zero (this element referred to achieve that $\left(x_{i}=x_{j}\right)$ )

That is $b_{i i}=0$ where $b_{i i}$ is called the main diagonal, $b_{i i}$ is an element in a matrix $M_{i j}$.

\subsubsection{An example showing how the dissimilarity matrix is obtained}

The following is an example for an information system, which consists of six projects; $X=\left\{p_{1}, p_{2}, p_{3}, p_{4}, p_{5}, p_{6}\right\}$ and three attributes; Funding, Poverty Impact and Middle Class Impact.

Table 1:shows the information system:

\begin{tabular}{|c|ccc|}
\hline \multicolumn{1}{|c|}{$\mathbf{U}$} & Funding & $\begin{array}{c}\text { Poverty } \\
\text { Impact }\end{array}$ & $\begin{array}{c}\text { Middle Class } \\
\text { Impact }\end{array}$ \\
\hline $\mathbf{p}_{\mathbf{1}}$ & High & High & Low \\
$\mathbf{p}_{\mathbf{2}}$ & Medium & low & High \\
$\mathbf{p}_{\mathbf{3}}$ & low & low & Medium \\
$\mathbf{p}_{\mathbf{4}}$ & low & Medium & Medium \\
$\mathbf{p}_{\mathbf{5}}$ & High & Medium & High \\
$\mathbf{p}_{\mathbf{6}}$ & High & High & High \\
\hline
\end{tabular}

To calculate the values of each element in the matrix using the law of dissimilarly matrix, we follow the following;

1- In attribute (Funding):

the number of iterations (High) in attribute funding column $=3$

the number of iterations (Medium) in attribute funding column $=1$

the number of iterations (Low) in attribute funding column $=2$

2- In attribute (Poverty Impact):

the number of iterations (High) in attribute poverty impact column $=2$

the number of iterations (Medium) in attribute poverty impact column $=2$

the number of iterations (Low) in attribute poverty impact column $=2$

3- In attribute (Middle Class Impact):

the number of iterations (High) in attribute middle class impact column $=3$

the number of iterations (Medium) in attribute middle class impact column $=2$

the number of iterations (Low) in attribute middle class impact column $=1$

1- We calculate $\left(m_{x i}, m_{y i}\right)=\left(p_{1}, p_{2}\right)$ : 
$[($ High, Medium), (High, Low $),($ Low, High $)]=\left[\frac{3+1}{3 \cdot 1} \cdot 1+\frac{2+2}{2 \cdot 2} \cdot 1+\frac{3+1}{3 \cdot 1} \cdot 1=\frac{4}{3}+\frac{4}{4}+\frac{4}{3}=\frac{11}{3}\right]$

2- We calculate $\left(m_{x i}, m_{y i}\right)=\left(p_{1}, p_{3}\right)$ :

$[($ High, Low $),($ High, Low $),($ Low, Medium $)]=\left[\frac{3+2}{3.2} \cdot 1+\frac{2+2}{2 \cdot 2} \cdot 1+\frac{2+1}{2 \cdot 1} \cdot 1=\frac{5}{6}+\frac{4}{4}+\frac{3}{2}=\frac{20}{6}=\frac{10}{3}\right]$

3- We calculate $\left(m_{x i}, m_{y i}\right)=\left(p_{1}, p_{4}\right)$ :

$\left[(\right.$ High, Low $),($ High, Medium), (Low, Medium) $]=\left[\frac{3+2}{3.2} \cdot 1+\frac{2+2}{2.2} \cdot 1+\frac{2+1}{2 \cdot 1} \cdot 1=\frac{5}{6}+\frac{4}{4}+\frac{3}{2}=\frac{11}{3}\right]$

4- We calculate $\left(m_{x i}, m_{y i}\right)=\left(p_{1}, p_{5}\right)$ :

$\left[(\right.$ High, High $),($ High, Medium), (Low, High) $]=\left[\frac{3+3}{3.3} \cdot 0+\frac{2+2}{2 \cdot 2} \cdot 1+\frac{3+1}{3 \cdot 1} \cdot 1=0+\frac{4}{4}+\frac{3}{2}=\frac{5}{2}\right]$

5- We calculate $\left(m_{x i}, m_{y i}\right)=\left(p_{1}, p_{6}\right)$ :

$[($ High, High $),($ High, High $),($ Low, High $)]=\left[\frac{3+3}{3.3} \cdot 0+\frac{2+2}{2.2} \cdot 0+\frac{3+1}{3.1} \cdot 1=0+0+\frac{4}{3}=\frac{4}{3}\right]$

Similarly, we obtain all elements in matrix.

Table2: shows the pretopolgy obtained from the information system

\begin{tabular}{|c|c|c|c|c|c|c|}
\hline & $x_{1}$ & $x_{2}$ & $x_{3}$ & $x_{4}$ & $x_{5}$ & $x_{6}$ \\
\hline$x_{1}$ & 0 & $\frac{11}{3}$ & $\frac{10}{3}$ & $\frac{11}{3}$ & $\frac{5}{2}$ & $\frac{4}{3}$ \\
\hline$x_{2}$ & $\frac{11}{3}$ & 0 & $\frac{7}{3}$ & $\frac{10}{3}$ & $\frac{7}{3}$ & $\frac{7}{3}$ \\
\hline$x_{3}$ & $\frac{10}{3}$ & $\frac{7}{3}$ & 0 & 1 & $\frac{8}{3}$ & $\frac{8}{3}$ \\
\hline$x_{4}$ & $\frac{11}{3}$ & $\frac{10}{3}$ & 1 & 0 & $\frac{8}{3}$ & $\frac{8}{3}$ \\
\hline$x_{5}$ & $\frac{5}{2}$ & $\frac{7}{3}$ & $\frac{8}{3}$ & $\frac{8}{3}$ & 0 & 1 \\
\hline$x_{6}$ & $\frac{4}{3}$ & $\frac{7}{3}$ & $\frac{8}{3}$ & $\frac{8}{3}$ & 1 & 0 \\
\hline
\end{tabular}

\section{Lemma 3.3.3}

When this matrix $M_{j}(x, y)$ is extracted, it is possible to create another matrix that shows the normalization that make the numbers in the matrix in the interval $[0,1]$.

\section{Remark 3.3.4}

In order to homogenize the distribution of the data, we normalize the data values by dividing the value of each element by the largest value in each column.

\subsection{Definition}

For $x \subset U$, approximation degree of accuracy ([1],[2]) for $X$ defined as;

$$
\text { Degree of Accuracy } \gamma_{x}=\frac{|\operatorname{int}(X)|}{\left|\zeta_{a}(X)\right|}
$$

where $|$.$| denotes the cardinality of a set.$ 


\subsection{The New Proposed Set of Reduction of Attributes}

When we find the degree of accuracy of the decision column, we follow the following:

\begin{tabular}{|c|l|}
\hline Case1 & $\begin{array}{l}\text { If the degree of accuracy after removing one attribute is higher than the degree of accuracy } \\
\text { for the original pretopology, then the existence of this attribute is unimportant, this attribute } \\
\text { is expendable. }\end{array}$ \\
\hline Case2 & $\begin{array}{l}\text { If the degree of accuracy after removing one attribute is less than the degree of accuracy for } \\
\text { the original pretopology, then this attribute is important and this attribute is indispensable. }\end{array}$ \\
\hline Case3 & $\begin{array}{l}\text { If the degree of accuracy after removing one attribute is the same as the degree of accuracy } \\
\text { for the original pretopology, then that attribute can be considered important when examining } \\
\text { a small set of attributes, but when we study a large set of attributes, we can say that this } \\
\text { attribute is unimportant. }\end{array}$ \\
\hline
\end{tabular}

\subsection{An Algorithm for the new proposed set}

The law of "Degree of Accuracy" is used to find the resulting pretopological space of all attributes, it also uses for finding the resulting pretopological space after removing an attribute, and then we compare "Degree of Accuracy" resulting from both the original pretopological space and the resulting after removing an attribute, then we will use the degree of accuracy to find the core in the information system.

\section{Algorithm Attribute Reduction Algorithm based on Measuring the Degree of Accuracy of Decision Sets In An Information System on Attributes.}

Input: an information system $I S=(U, Q, V)$, where $U=\left\{x_{1}, x_{2}, x_{3}, \ldots, x_{n}\right\} ., Q$ is a set of attributes and $\mathrm{V}$ is an attribute value set where $V=\left\{v_{0}, v_{1}, v_{3}, \ldots, v_{n}\right\}$.

$/ / V_{0}$ denotes the first attribute.

Output: An attribute reduction of this information system.

Step 1 Create a dissimilarity matrix $|U| \times|U|$.

Step2 Calculate a matrix elements using,

$$
M_{i j}(x, y)=\sum_{i=1}^{n} \sum_{j=1}^{n} \frac{\left(m_{x i}+m_{y i}\right)}{m_{x i} m_{y i}} \delta\left(x_{i}, y_{j}\right)
$$

where $\delta\left(x_{i}, x_{j}\right)=\left\{\begin{array}{lll}0 & \text { if } & \left(x_{i}=x_{j}\right) \\ 1 & \text { if } & \left(x_{i} \neq x_{j}\right)\end{array}\right.$

Step 3 If $\left(x_{i}=x_{j}\right)$, then put $b_{i i}=0, i \in[1, n]$.

Step 4 Normalization of dissimilarity matrix to make the numbers in the interval $[0,1]$.

Step 5 Calculate

$$
\zeta_{a}=X_{i} \cup\left\{X \in U / \mu\left(X_{i}, X_{j} \geq \delta\right), 1 \geq \delta \geq 0, i, j \in[1, n], n=|U|\right\}
$$

Step 6 Calculate $\operatorname{int}_{\zeta_{a}}\left(x_{i}\right)=\operatorname{co}\left(\zeta_{a}\left(\operatorname{cox}_{i}\right)\right), i \in[1, n]$.

Step 7 For each attribute $V \in Q$, Update the attribute $Q=Q-\left\{V_{0}\right\}$, and then go to Step 1.

Step 8 For each product pretopological space, Calculate $\gamma_{x}=\frac{\left|\operatorname{int}\left(x_{i}\right)\right|}{\left|\zeta_{a}\left(x_{i}\right)\right|}$ "Degree of Accuracy"

Step 9 If degree of accuracy after removing one attribute $>$ degree of accuracy for the original pretopology, then this attribute is unimportant.

If degree of accuracy after removing one attribute $<$ degree of accuracy for the original pretopology, then this attribute is important.

If degree of accuracy after removing one attribute $=$ degree of accuracy for the original pretopology, then that attribute can be considered important when examining a small set of attributes, but when we study a large set of attributes, we can say that this attribute is unimportant, then the algorithm terminates. 
The following is an example for information system:-

\section{AN ILLUSTRATIVE EXAMPLE SHOWING THE METHOD OF DISSIMILARITY}

we give an example from information system, which consists of eight applied for job; $X=\left\{x_{1}, x_{2}, x_{3}, x_{4}, x_{5}, x_{6}, x_{7}, x_{8}\right\}$ and four attributes; Diploma, French, Experience and Reference.

Table 3:shows the information system:

\begin{tabular}{|c|ccccc|}
\multicolumn{1}{c}{$\mathbf{U}$} & Diploma & French & Experience & Reference & Decision \\
\hline $\mathbf{x}_{\mathbf{1}}$ & MBA & Yes & Medium & Excellent & Accept \\
$\mathbf{x}_{\mathbf{2}}$ & MBA & Yes & Low & Neutral & Reject \\
$\mathbf{x}_{\mathbf{3}}$ & MCE & Yes & Low & Good & Reject \\
$\mathbf{x}_{\mathbf{4}}$ & MSc & Yes & High & Neutral & Accept \\
$\mathbf{x}_{\mathbf{5}}$ & MSc & Yes & Medium & Neutral & Reject \\
$\mathbf{x}_{\mathbf{6}}$ & MSc & Yes & High & Excellent & Reject \\
$\mathbf{x}_{\mathbf{7}}$ & MBA & No & High & Good & Accept \\
$\mathbf{x}_{\mathbf{8}}$ & MCE & No & Low & Excellent & Reject \\
\hline
\end{tabular}

Where MBA denoted Master of Business Administration, MCE denoted Microsoft Certified Educator, MSc denoted Master of Science.

\subsection{Create a pretopology from an information system using a matrix}

For the example given in section 4, we propose the following methodology:

Firstly, we generate pretopological space from information system by using (Definition 3.2) in order to find the following matrices which give with the relation a dissimilarity method.

Secondly, use the following relation to find a pretopology for the attributes; denoted by $\zeta_{\mathrm{a}}$ :

$$
\zeta_{a} x_{i}=x_{i} \cup\left\{\mu\left(x_{i}, x_{j}\right)=0.5 \quad i, j=1,2,3, \ldots, 8\right\} \quad \text { Eq. (1) }
$$

Thirdly, we calculate the degree of accuracy using the decision column, so we break the decision column into two parts :

$\{\text { Decision }\}_{\text {Accept }}=\left\{\mathrm{x}_{1}, \mathrm{x}_{4}, \mathrm{x}_{7}\right\}$

$\{\text { Decision }\}_{\text {Reject }}=\left\{\mathrm{x}_{2}, \mathrm{x}_{3}, \mathrm{x}_{5}, \mathrm{x}_{6}, \mathrm{x}_{8}\right\}$

Then,

1) We calculate:

1. $\zeta_{\mathrm{a}}\{\text { Decision }\}_{\text {Accept }}$ and int $_{\zeta_{\mathrm{a}}}\{\text { Decision }\}_{\text {Accept }}$ using (Definition 2.2).

2. $\zeta_{\mathrm{a}}\{\text { Decision }\}_{\text {Reject }}$ and int $_{\zeta_{\mathrm{a}}}\{\text { Decision }\}_{\text {Reject }}$ using (Definition 2.2).

2) We calculate the degree of accuracy using (Definition 3.3) for each $\{\text { Decision }\}_{\text {Accept }},\{\text { Decision }\}_{\text {Reject }}$

\subsubsection{Constructing a pretopology from information system using four attributes}

To commence, pretopologies resulting from the four attributes are to be obtained as follows: for each pretopology an $8 * 8$ matrix is to be constructed where each element along the main diagonal is equal zero; while each element of the remaining elements of the matrix is to be use the relation dissimilarity method (Definition 3.2) 
Table 4: shows the pretopology obtained from the information system.

\begin{tabular}{|l|c|c|c|c|c|c|c|c|}
\hline & $x_{1}$ & $x_{2}$ & $x_{3}$ & $x_{4}$ & $x_{5}$ & $x_{6}$ & $x_{7}$ & $x_{8}$ \\
\hline$x_{1}$ & 0 & $\frac{3}{2}$ & $\frac{5}{2}$ & $\frac{3}{2}$ & $\frac{4}{3}$ & $\frac{3}{2}$ & $\frac{7}{3}$ & $\frac{7}{3}$ \\
\hline$x_{2}$ & $\frac{3}{2}$ & 0 & $\frac{5}{3}$ & $\frac{4}{3}$ & $\frac{3}{2}$ & 2 & $\frac{7}{3}$ & $\frac{13}{6}$ \\
\hline$x_{3}$ & $\frac{5}{2}$ & $\frac{5}{3}$ & 0 & $\frac{7}{3}$ & $\frac{5}{2}$ & $\frac{7}{3}$ & $\frac{13}{6}$ & $\frac{3}{2}$ \\
\hline$x_{4}$ & $\frac{3}{2}$ & $\frac{4}{3}$ & $\frac{7}{3}$ & 0 & $\frac{5}{6}$ & $\frac{2}{3}$ & $\frac{13}{6}$ & $\frac{17}{6}$ \\
\hline$x_{5}$ & $\frac{4}{3}$ & $\frac{3}{2}$ & $\frac{5}{2}$ & $\frac{5}{6}$ & 0 & $\frac{3}{2}$ & $\frac{3}{3}$ & $\frac{3}{3}$ \\
\hline$x_{6}$ & $\frac{3}{2}$ & 2 & $\frac{7}{3}$ & $\frac{2}{3}$ & $\frac{3}{2}$ & 0 & $\frac{13}{6}$ & $\frac{13}{6}$ \\
\hline$x_{7}$ & $\frac{7}{3}$ & $\frac{7}{3}$ & $\frac{13}{6}$ & $\frac{13}{6}$ & 3 & $\frac{13}{6}$ & 0 & $\frac{7}{3}$ \\
\hline$x_{8}$ & $\frac{7}{3}$ & $\frac{13}{6}$ & $\frac{3}{2}$ & $\frac{17}{6}$ & 3 & $\frac{13}{6}$ & $\frac{7}{3}$ & 0 \\
\hline
\end{tabular}

Table 5: shows the normalization to make the numbers in the interval $[0,1]$ obtained from the information system.

\begin{tabular}{|l|l|l|l|l|l|l|l|l|}
\hline & $x_{1}$ & $x_{2}$ & $x_{3}$ & $x_{4}$ & $x_{5}$ & $x_{6}$ & $x_{7}$ & $x_{8}$ \\
\hline$x_{1}$ & 0 & 0.6 & 1 & 0.5 & 0.4 & 0.6 & 0.7 & 0.7 \\
\hline$x_{2}$ & 0.6 & 0 & 0.6 & 0.4 & 0.5 & 0.8 & 0.7 & 0.7 \\
\hline$x_{3}$ & 1 & 0.7 & 0 & 0.8 & 0.8 & 1 & 0.7 & 0.5 \\
\hline$x_{4}$ & 0.6 & 0.5 & 0.9 & 0 & 0.2 & 0.2 & 0.7 & 0.9 \\
\hline$x_{5}$ & 0.5 & 0.6 & 1 & 0.2 & 0 & 0.6 & 1 & 1 \\
\hline$x_{6}$ & 0.6 & 0.8 & 0.9 & 0.2 & 0.5 & 0 & 0.7 & 0.7 \\
\hline$x_{7}$ & 0.9 & 1 & 0.8 & 0.7 & 1 & 0.9 & 0 & 0.7 \\
\hline$x_{8}$ & 0.9 & 0.9 & 0.6 & 1 & 1 & 0.9 & 0.7 & 0 \\
\hline
\end{tabular}

Therefore, the pretopology component of matrix by using Eq. (1)

$\zeta_{a_{1}}\left\{x_{1}\right\}=\left\{x_{1}, x_{4}\right\}$

$\zeta_{a_{1}}\left\{x_{2}\right\}=\left\{x_{2}, x_{5}\right\}$

$\zeta_{a_{1}}\left\{x_{3}\right\}=\left\{x_{3}, x_{8}\right\}$

$\zeta_{a_{1}}\left\{x_{4}\right\}=\left\{x_{2}, x_{4}\right\}$

$\zeta_{a_{1}}\left\{x_{5}\right\}=\left\{x_{1}, x_{5}\right\}$

$\zeta_{a_{1}}\left\{x_{6}\right\}=\left\{x_{5}, x_{6}\right\}$

$\zeta_{a_{1}}\left\{x_{7}\right\}=\left\{x_{7}\right\}$

$\zeta_{a_{1}}\left\{x_{8}\right\}=\left\{x_{8}\right\}$

Therefore,

1- Calculate $\zeta_{a}\{\text { Decision }\}_{\text {Accept }}$ and $\operatorname{int}_{\zeta_{a}}\{\text { Decision }\}_{\text {Accept }}$

\begin{tabular}{|c|c|}
\hline$\zeta_{a}\{\text { Decision }\}_{\text {Accept }}$ & $\zeta_{a}\left\{x_{1}, x_{4}, x_{7}\right\}=\left\{x_{1}, x_{2}, x_{4}, x_{7}\right\}$ \\
\hline $\operatorname{int}_{\zeta_{a}}\{\text { Decision }\}_{\text {Accept }}$ & $\begin{array}{c}\operatorname{int}_{\zeta_{a}}\left\{x_{1}, x_{4}, x_{7}\right\}=\operatorname{co}\left\{\zeta_{a} \operatorname{co}\left\{x_{1}, x_{4}, x_{7}\right\}\right\}=\operatorname{co}\left\{\zeta_{a}\left\{x_{2}, x_{3}, x_{5}, x_{6}, x_{8}\right\}\right\} \\
=\operatorname{co}\left\{x_{1}, x_{2}, x_{3}, x_{5}, x_{6}, x_{8}\right\}=\left\{x_{4}, x_{7}\right\}\end{array}$ \\
\hline Degree of Accuracy & $\frac{\left|x_{4}, x_{7}\right|}{\left|x_{1}, x_{2}, x_{4}, x_{7}\right|}=\frac{2}{4}=0.5$ \\
\hline
\end{tabular}


2- calculate $\zeta_{a}\{\text { Decision }\}_{\text {Reject }}$ and int $_{\zeta_{a}}\{\text { Decision }\}_{\text {Reject }}$

\begin{tabular}{|c|c|}
\hline$\zeta_{a}\{\text { Decision }\}_{\text {Reject }}$ & $\zeta_{a}\left\{x_{2}, x_{3}, x_{5}, x_{6}, x_{8}\right\}=\left\{x_{1}, x_{2}, x_{3}, x_{5}, x_{6}, x_{8}\right\}$ \\
\hline int $_{\zeta_{a}}\{\text { Decision }\}_{\text {Reject }}$ & $\begin{array}{c}\text { int }_{\zeta_{a}}\left\{x_{2}, x_{3}, x_{5}, x_{6}, x_{8}\right\}=\operatorname{co}\left\{\zeta_{a}\left\{x_{2}, x_{3}, x_{5}, x_{6}, x_{8}\right\}\right\}=\operatorname{co}\left\{\zeta_{a}\left\{x_{1}, x_{4}, x_{7}\right\}\right\} \\
=\operatorname{co}\left\{x_{1}, x_{2}, x_{4}, x_{7}\right\}=\left\{x_{3}, x_{5}, x_{6}, x_{8}\right\}\end{array}$ \\
\hline Degree of Accuracy & $\frac{\left|x_{3}, x_{5}, x_{6}, x_{8}\right|}{\left|x_{1}, x_{2}, x_{3}, x_{5}, x_{6}, x_{8}\right|}=\frac{4}{6}=0.6$ \\
\hline
\end{tabular}

\subsubsection{Constructing a pretopology when removing the attribute Diploma}

Table 6: shows the pretopology obtained when Table 7: shows the normalization to make the numbers removing the attribute diploma. in the interval $[0,1]$ obtained from the information system.

\begin{tabular}{|l|l|l|l|l|l|l|l|l|}
\hline & $x_{1}$ & $x_{2}$ & $x_{3}$ & $x_{4}$ & $x_{5}$ & $x_{6}$ & $x_{7}$ & $x_{8}$ \\
\hline$x_{1}$ & 0 & $\frac{3}{2}$ & $\frac{5}{2}$ & $\frac{3}{2}$ & $\frac{2}{3}$ & $\frac{5}{6}$ & $\frac{7}{3}$ & $\frac{3}{2}$ \\
\hline$x_{2}$ & $\frac{3}{2}$ & 0 & $\frac{5}{6}$ & $\frac{2}{3}$ & $\frac{5}{6}$ & $\frac{4}{3}$ & $\frac{7}{3}$ & $\frac{4}{3}$ \\
\hline$x_{3}$ & $\frac{5}{3}$ & $\frac{5}{6}$ & 0 & $\frac{3}{2}$ & $\frac{5}{3}$ & $\frac{3}{2}$ & $\frac{4}{3}$ & $\frac{3}{2}$ \\
\hline$x_{4}$ & $\frac{3}{2}$ & $\frac{2}{3}$ & $\frac{3}{2}$ & 0 & $\frac{5}{6}$ & $\frac{2}{3}$ & $\frac{3}{2}$ & $\frac{2}{3}$ \\
\hline$x_{5}$ & $\frac{2}{3}$ & $\frac{5}{6}$ & $\frac{5}{3}$ & $\frac{5}{6}$ & 0 & $\frac{3}{2}$ & $\frac{7}{3}$ & $\frac{13}{6}$ \\
\hline$x_{6}$ & $\frac{5}{6}$ & $\frac{4}{3}$ & $\frac{3}{2}$ & $\frac{2}{3}$ & $\frac{3}{2}$ & 0 & $\frac{3}{2}$ & $\frac{4}{3}$ \\
\hline$x_{7}$ & $\frac{7}{3}$ & $\frac{7}{3}$ & $\frac{4}{3}$ & $\frac{3}{2}$ & $\frac{7}{3}$ & $\frac{3}{2}$ & 0 & $\frac{3}{2}$ \\
\hline$x_{8}$ & $\frac{3}{2}$ & $\frac{4}{3}$ & $\frac{3}{2}$ & 2 & $\frac{13}{6}$ & $\frac{4}{3}$ & $\frac{3}{2}$ & 0 \\
\hline
\end{tabular}

\begin{tabular}{|l|l|l|l|l|l|l|l|l|}
\hline & $x_{1}$ & $x_{2}$ & $x_{3}$ & $x_{4}$ & $x_{5}$ & $x_{6}$ & $x_{7}$ & $x_{8}$ \\
\hline$x_{1}$ & 0 & 0.6 & 1 & 0.7 & 0.2 & 0.5 & 1 & 0.6 \\
\hline$x_{2}$ & 0.6 & 0 & 0.5 & 0.3 & 0.3 & 0.8 & 1 & 0.6 \\
\hline$x_{3}$ & 0.7 & 0.3 & 0 & 0.7 & 0.7 & 1 & 0.5 & 0.6 \\
\hline$x_{4}$ & 0.6 & 0.2 & 0.9 & 0 & 0.3 & 0.4 & 0.6 & 0.9 \\
\hline$x_{5}$ & 0.2 & 0.3 & 1 & 0.4 & 0 & 1 & 1 & 1 \\
\hline$x_{6}$ & 0.3 & 0.5 & 0.9 & 0.3 & 0.6 & 0 & 0.6 & 0.6 \\
\hline$x_{7}$ & 1 & 1 & 0.8 & 0.7 & 1 & 1 & 0 & 0.6 \\
\hline$x_{8}$ & 0.6 & 0.5 & 0.9 & 1 & 0.9 & 0.8 & 0.6 & 0 \\
\hline
\end{tabular}

Therefore, the pretopology component of matrix by using Eq. (1)

$$
\begin{aligned}
& \zeta_{a_{2}}\left\{x_{1}\right\}=\left\{x_{1}, x_{6}\right\} \\
& \zeta_{a_{2}}\left\{x_{2}\right\}=\left\{x_{2}, x_{3}\right\} \\
& \zeta_{a_{2}}\left\{x_{3}\right\}=\left\{x_{3}, x_{7}\right\} \\
& \zeta_{a_{2}}\left\{x_{4}\right\}=\left\{x_{4}\right\} \\
& \zeta_{a_{2}}\left\{x_{5}\right\}=\left\{x_{5}\right\} \\
& \zeta_{a_{2}}\left\{x_{6}\right\}=\left\{x_{2}, x_{6}\right\} \\
& \zeta_{a_{2}}\left\{x_{7}\right\}=\left\{x_{7}\right\} \\
& \zeta_{a_{2}}\left\{x_{8}\right\}=\left\{x_{2}, x_{8}\right\}
\end{aligned}
$$




\section{Therefore,}

1- Calculate $\zeta_{a}\{\text { Decision }\}_{\text {Accept }}$ and int $_{\zeta_{a}}\{\text { Decision }\}_{\text {Accept }}$

\begin{tabular}{|l|c|}
\hline $\multicolumn{1}{|c|}{\text { Decision }}_{\text {Accept }}$ & $\zeta_{a}\left\{x_{1}, x_{4}, x_{7}\right\}=\left\{x_{1}, x_{4}, x_{6}, x_{7}\right\}$ \\
\hline int $_{\zeta_{a}}\{\text { Decision }\}_{\text {Accept }}$ & int $_{\zeta_{a}}\left\{x_{1}, x_{4}, x_{7}\right\}=\operatorname{co}\left\{\zeta_{a} \operatorname{co}\left\{x_{1}, x_{4}, x_{7}\right\}\right\}=\operatorname{co}\left\{\zeta_{a}\left\{x_{2}, x_{3}, x_{5}, x_{6}, x_{8}\right\}\right\}$ \\
$=$ & $\operatorname{co}\left\{x_{2}, x_{3}, x_{5}, x_{6}, x_{7}, x_{8}\right\}=\left\{x_{1}, x_{4}\right\}$
\end{tabular}

2- calculate $\zeta_{a}\{\text { Decision }\}_{\text {Reject }}$ and int $_{\zeta_{a}}\{\text { Decision }\}_{\text {Reject }}$

\begin{tabular}{|l|c|}
\hline$\zeta_{a}\{\text { Decision }\}_{\text {Reject }}$ & $\zeta_{a}\left\{x_{2}, x_{3}, x_{5}, x_{6}, x_{8}\right\}=\left\{x_{2}, x_{3}, x_{5}, x_{6}, x_{7}, x_{8}\right\}$ \\
\hline int $_{\zeta_{a}}\{\text { Decision }\}_{\text {Reject }}$ & $\begin{array}{c}\text { int }_{\zeta_{a}}\left\{x_{2}, x_{3}, x_{5}, x_{6}, x_{8}\right\}=\operatorname{co}\left\{\zeta_{a}\left\{x_{2}, x_{3}, x_{5}, x_{6}, x_{8}\right\}\right\}=\operatorname{co}\left\{\zeta_{a}\left\{x_{1}, x_{4}, x_{7}\right\}\right\} \\
=\operatorname{co}\left\{x_{1}, x_{4}, x_{6}, x_{7}\right\}=\operatorname{co}\left\{x_{2}, x_{3}, x_{5}, x_{8}\right\}\end{array}$ \\
\hline Degree of Accuracy & $\frac{\left|x_{2}, x_{3}, x_{5}, x_{8}\right|}{\left|x_{2}, x_{3}, x_{5}, x_{6}, x_{7}, x_{8}\right|}=\frac{4}{6}=0.6$ \\
\hline
\end{tabular}

\subsubsection{Constructing a pretopology when removing the attribute French}

Table 8: shows the pretopology obtained when removing the attribute french.

\begin{tabular}{|l|c|c|c|c|c|c|c|c|}
\hline & $x_{1}$ & $x_{2}$ & $x_{3}$ & $x_{4}$ & $x_{5}$ & $x_{6}$ & $x_{7}$ & $x_{8}$ \\
\hline$x_{1}$ & 0 & $\frac{3}{2}$ & $\frac{5}{2}$ & $\frac{3}{2}$ & $\frac{4}{3}$ & $\frac{3}{2}$ & $\frac{5}{3}$ & $\frac{5}{3}$ \\
\hline$x_{2}$ & $\frac{3}{2}$ & 0 & $\frac{5}{3}$ & $\frac{4}{3}$ & $\frac{3}{2}$ & 2 & $\frac{5}{3}$ & $\frac{3}{2}$ \\
\hline$x_{3}$ & $\frac{5}{2}$ & $\frac{5}{3}$ & 0 & $\frac{7}{3}$ & $\frac{5}{2}$ & $\frac{7}{3}$ & $\frac{13}{6}$ & $\frac{5}{6}$ \\
\hline$x_{4}$ & $\frac{3}{2}$ & $\frac{4}{3}$ & $\frac{7}{3}$ & 0 & $\frac{5}{6}$ & $\frac{2}{3}$ & $\frac{3}{2}$ & $\frac{13}{6}$ \\
\hline$x_{5}$ & $\frac{4}{3}$ & $\frac{3}{2}$ & $\frac{5}{2}$ & $\frac{5}{6}$ & 0 & $\frac{3}{2}$ & $\frac{7}{3}$ & $\frac{7}{3}$ \\
\hline$x_{6}$ & $\frac{3}{2}$ & 2 & $\frac{7}{3}$ & $\frac{2}{3}$ & $\frac{3}{2}$ & 0 & $\frac{3}{2}$ & $\frac{3}{2}$ \\
\hline$x_{7}$ & $\frac{5}{3}$ & $\frac{5}{3}$ & $\frac{13}{6}$ & $\frac{3}{2}$ & $\frac{7}{3}$ & $\frac{3}{2}$ & 0 & $\frac{7}{3}$ \\
\hline$x_{8}$ & $\frac{5}{3}$ & $\frac{3}{2}$ & $\frac{5}{6}$ & $\frac{13}{6}$ & $\frac{7}{3}$ & $\frac{3}{2}$ & $\frac{7}{3}$ & 0 \\
\hline
\end{tabular}

Table 9: shows the normalization to make the numbers in the interval $[0,1]$ obtained from the information system.

\begin{tabular}{|l|l|l|l|l|l|l|l|l|}
\hline & $\boldsymbol{x}_{1}$ & $\boldsymbol{x}_{2}$ & $\boldsymbol{x}_{3}$ & $\boldsymbol{x}_{4}$ & $\boldsymbol{x}_{5}$ & $\boldsymbol{x}_{6}$ & $\boldsymbol{x}_{7}$ & $\boldsymbol{x}_{8}$ \\
\hline$x_{1}$ & 0 & 0.7 & 1 & 0.6 & 0.5 & 0.6 & 0.7 & 0.7 \\
\hline $\boldsymbol{x}_{2}$ & 0.6 & 0 & 0.6 & 0.5 & 0.6 & 1 & 0.7 & 0.6 \\
\hline $\boldsymbol{x}_{3}$ & 1 & 0.8 & 0 & 1 & 1 & 1 & 0.9 & 0.3 \\
\hline $\boldsymbol{x}_{4}$ & 0.6 & 0.6 & 0.9 & 0 & 0.3 & 0.8 & 0.6 & 0.9 \\
\hline $\boldsymbol{x}_{5}$ & 0.5 & 0.7 & 1 & 0.3 & 0 & 0.6 & 1 & 1 \\
\hline $\boldsymbol{x}_{6}$ & 0.6 & 1 & 0.9 & 0.2 & 0.6 & 0 & 0.6 & 0.6 \\
\hline $\boldsymbol{x}_{7}$ & 0.6 & 0.8 & 0.8 & 0.6 & 0.9 & 0.6 & 0 & 1 \\
\hline $\boldsymbol{x}_{8}$ & 0.6 & 0.7 & 0.3 & 0.9 & 0.9 & 0.6 & 1 & 0 \\
\hline
\end{tabular}


Therefore, the pretopology component of matrix by using Eq. (1)

$$
\begin{aligned}
& \zeta_{a_{3}}\left\{x_{1}\right\}=\left\{x_{1}, x_{5}\right\} \\
& \zeta_{a_{3}}\left\{x_{2}\right\}=\left\{x_{2}, x_{4}\right\} \\
& \zeta_{a_{3}}\left\{x_{3}\right\}=\left\{x_{3}\right\} \\
& \zeta_{a_{3}}\left\{x_{4}\right\}=\left\{x_{4}\right\} \\
& \zeta_{a_{3}}\left\{x_{5}\right\}=\left\{x_{1}, x_{5}\right\} \\
& \zeta_{a_{3}}\left\{x_{6}\right\}=\left\{x_{6}\right\} \\
& \zeta_{a_{3}}\left\{x_{7}\right\}=\left\{x_{7}\right\} \\
& \zeta_{a_{3}}\left\{x_{8}\right\}=\left\{x_{8}\right\}
\end{aligned}
$$

\begin{tabular}{|c|c|}
\hline$\zeta_{a}\{\text { Decision }\}_{\text {Accept }}$ & $\zeta_{a}\left\{x_{1}, x_{4}, x_{7}\right\}=\left\{x_{1}, x_{4}, x_{5}, x_{7}\right\}$ \\
\hline int $_{\zeta_{a}}\{\text { Decision }\}_{\text {Accept }}$ & $\begin{aligned} \operatorname{int}_{\zeta_{a}}\left\{x_{1}, x_{4}, x_{7}\right\} & =\operatorname{co}\left\{\zeta_{a} \operatorname{co}\left\{x_{1}, x_{4}, x_{7}\right\}\right\}=\operatorname{co}\left\{\zeta_{a}\left\{x_{2}, x_{3}, x_{5}, x_{6}, x_{8}\right\}\right\} \\
& =\operatorname{co}\left\{x_{1}, x_{2}, x_{3}, x_{4}, x_{5}, x_{6}, x_{8}\right\}=\left\{x_{7}\right\}\end{aligned}$ \\
\hline Degree of Accuracy & $\frac{\left|x_{7}\right|}{\left|x_{1}, x_{4}, x_{5}, x_{7}\right|}=\frac{1}{4}=0.25$ \\
\hline
\end{tabular}

\begin{tabular}{|c|c|}
\hline$\zeta_{a}\{\text { Decision }\}_{\text {Reject }}$ & $\zeta_{a}\left\{x_{2}, x_{3}, x_{5}, x_{6}, x_{8}\right\}=\left\{x_{1}, x_{2}, x_{3}, x_{4}, x_{5}, x_{6}, x_{8}\right\}$ \\
\hline int $_{\zeta_{a}}\{\text { Decision }\}_{\text {Reject }}$ & $\begin{array}{c}\operatorname{int}_{\zeta_{a}}\left\{x_{2}, x_{3}, x_{5}, x_{6}, x_{8}\right\}=\operatorname{co}\left\{\zeta_{a}\left\{x_{2}, x_{3}, x_{5}, x_{6}, x_{8}\right\}\right\}=\operatorname{co}\left\{\zeta_{a}\left\{x_{1}, x_{4}, x_{7}\right\}\right\} \\
=\operatorname{co}\left\{x_{1}, x_{4}, x_{5}, x_{7}\right\}=\operatorname{co}\left\{x_{2}, x_{3}, x_{6}, x_{8}\right\}\end{array}$ \\
\hline Degree of Accuracy & $\frac{\left|x_{2}, x_{3}, x_{6}, x_{8}\right|}{\left|x_{1}, x_{2}, x_{3}, x_{4}, x_{5}, x_{6}, x_{8}\right|}=\frac{4}{7}=0.57$ \\
\hline
\end{tabular}

\section{Therefore,}

1- Calculate $\zeta_{a}\{\text { Decision }\}_{\text {Accept }}$ and $\operatorname{int}_{\zeta_{a}}\{\text { Decision }\}_{\text {Accept }}$

2- calculate $\zeta_{a}\{\text { Decision }\}_{\text {Reject }}$ and int $_{\zeta_{a}}\{\text { Decision }\}_{\text {Reject }}$ 


\subsubsection{Constructing a pretopology when removing the attribute Experience}

Table 10: shows the pretopology obtained when removing the attribute experience.
Table 11: shows the normalization to make the numbersin the interval $[0,1]$ obtained from the information system.

\begin{tabular}{|l|c|c|c|c|c|c|c|c|}
\hline & $x_{1}$ & $x_{2}$ & $x_{3}$ & $x_{4}$ & $x_{5}$ & $x_{6}$ & $x_{7}$ & $x_{8}$ \\
\hline$x_{1}$ & 0 & $\frac{2}{3}$ & $\frac{5}{3}$ & $\frac{4}{3}$ & $\frac{4}{3}$ & $\frac{2}{3}$ & $\frac{3}{2}$ & $\frac{3}{2}$ \\
\hline$x_{2}$ & $\frac{2}{3}$ & 0 & $\frac{5}{3}$ & $\frac{2}{3}$ & $\frac{2}{3}$ & $\frac{4}{3}$ & $\frac{3}{2}$ & $\frac{13}{6}$ \\
\hline$x_{3}$ & $\frac{5}{3}$ & $\frac{5}{3}$ & 0 & $\frac{5}{3}$ & $\frac{5}{3}$ & $\frac{5}{3}$ & $\frac{3}{2}$ & $\frac{3}{2}$ \\
\hline$x_{4}$ & $\frac{4}{3}$ & $\frac{2}{3}$ & $\frac{5}{3}$ & 0 & 0 & $\frac{2}{3}$ & $\frac{13}{6}$ & $\frac{13}{6}$ \\
\hline$x_{5}$ & $\frac{4}{3}$ & $\frac{2}{3}$ & $\frac{5}{3}$ & 0 & 0 & $\frac{2}{3}$ & $\frac{13}{6}$ & $\frac{13}{6}$ \\
\hline$x_{6}$ & $\frac{2}{3}$ & $\frac{4}{3}$ & $\frac{5}{3}$ & $\frac{2}{3}$ & $\frac{2}{3}$ & 0 & $\frac{13}{6}$ & $\frac{3}{2}$ \\
\hline$x_{7}$ & $\frac{3}{2}$ & $\frac{3}{2}$ & $\frac{3}{2}$ & $\frac{13}{6}$ & $\frac{13}{6}$ & $\frac{13}{6}$ & 0 & $\frac{5}{3}$ \\
\hline$x_{8}$ & $\frac{3}{2}$ & $\frac{13}{6}$ & $\frac{3}{2}$ & $\frac{13}{6}$ & $\frac{13}{6}$ & $\frac{3}{2}$ & $\frac{5}{3}$ & 0 \\
\hline
\end{tabular}

\begin{tabular}{|l|l|l|l|l|l|l|l|l|}
\hline & $x_{1}$ & $\boldsymbol{x}_{2}$ & $\boldsymbol{x}_{3}$ & $\boldsymbol{x}_{4}$ & $\boldsymbol{x}_{5}$ & $\boldsymbol{x}_{6}$ & $\boldsymbol{x}_{7}$ & $\boldsymbol{x}_{8}$ \\
\hline$x_{1}$ & 0 & 0.3 & 1 & 0.6 & 0.6 & 0.3 & 0.6 & 0.6 \\
\hline $\boldsymbol{x}_{2}$ & 0.4 & 0 & 1 & 0.3 & 0.3 & 0.6 & 0.6 & 1 \\
\hline $\boldsymbol{x}_{3}$ & 1 & 0.7 & 0 & 0.7 & 0.7 & 0.7 & 0.6 & 0.6 \\
\hline $\boldsymbol{x}_{4}$ & 0.8 & 0.3 & 1 & 0 & 0 & 0.3 & 1 & 1 \\
\hline $\boldsymbol{x}_{5}$ & 0.8 & 0.3 & 1 & 0 & 0 & 0.3 & 1 & 1 \\
\hline $\boldsymbol{x}_{6}$ & 0.4 & 0.6 & 1 & 0.3 & 0.3 & 0 & 1 & 0.6 \\
\hline $\boldsymbol{x}_{7}$ & 0.9 & 0.6 & 0.9 & 1 & 1 & 1 & 0 & 0.7 \\
\hline $\boldsymbol{x}_{8}$ & 0.9 & 1 & 0.9 & 1 & 1 & 0.6 & 0.7 & 0 \\
\hline
\end{tabular}

Therefore, the pretopology component of matrix by using Eq. (1)

$\zeta_{a_{4}}\left\{x_{1}\right\}=\left\{x_{1}\right\}$

$\zeta_{a_{4}}\left\{x_{2}\right\}=\left\{x_{2}\right\}$

$\zeta_{a_{4}}\left\{x_{3}\right\}=\left\{x_{3}\right\}$

$\zeta_{a_{4}}\left\{x_{4}\right\}=\left\{x_{4}\right\}$

$\zeta_{a_{4}}\left\{x_{5}\right\}=\left\{x_{5}\right\}$

$\zeta_{a_{4}}\left\{x_{6}\right\}=\left\{x_{6}\right\}$

$\zeta_{a_{4}}\left\{x_{7}\right\}=\left\{x_{7}\right\}$

$\zeta_{a_{4}}\left\{x_{8}\right\}=\left\{x_{8}\right\}$

\section{Therefore,}

1- Calculate $\zeta_{a}\{\text { Decision }\}_{\text {Accept }}$ and int $_{\zeta_{a}}\{\text { Decision }\}_{\text {Accept }}$

\begin{tabular}{|l|c|}
\hline \multicolumn{1}{|c|}{\begin{tabular}{c|}
$\zeta_{a}\{\text { Decision }\}_{\text {Accept }}\left\{x_{1}, x_{4}, x_{7}\right\}=\left\{x_{1}, x_{4}, x_{7}\right\}$ \\
\hline int $_{\zeta_{a}}\{\text { Decision }\}_{\text {Accept }}$
\end{tabular}} & $\begin{array}{r}\text { int }_{\zeta_{a}}\left\{x_{1}, x_{4}, x_{7}\right\}=\operatorname{co}\left\{\zeta_{a} \operatorname{co}\left\{x_{1}, x_{4}, x_{7}\right\}\right\}=\operatorname{co}\left\{\zeta_{a}\left\{x_{2}, x_{3}, x_{5}, x_{6}, x_{8}\right\}\right\} \\
=\operatorname{co}\left\{x_{2}, x_{3}, x_{5}, x_{6}, x_{8}\right\}=\left\{x_{1}, x_{4}, x_{7}\right\}\end{array}$ \\
\hline Degree of Accuracy & $\frac{\left|x_{1}, x_{4}, x_{7}\right|}{\left|x_{1}, x_{4}, x_{7}\right|}=\frac{3}{3}=1$ \\
\hline
\end{tabular}


calculate $\zeta_{a}\{\text { Decision }\}_{\text {Reject }}$ and int $_{\zeta_{a}}\{\text { Decision }\}_{\text {Reject }}$

\begin{tabular}{|c|c|}
\hline$\zeta_{a}\{\text { Decision }\}_{\text {Reject }}$ & $\zeta_{a}\left\{x_{2}, x_{3}, x_{5}, x_{6}, x_{8}\right\}=\left\{x_{2}, x_{3}, x_{5}, x_{6}, x_{8}\right\}$ \\
\hline int $_{\zeta_{a}}\{\text { Decision }\}_{\text {Reject }}$ & $\begin{array}{c}\text { int }_{\zeta_{a}}\left\{x_{2}, x_{3}, x_{5}, x_{6}, x_{8}\right\}=\operatorname{co}\left\{\zeta_{a}\left\{x_{2}, x_{3}, x_{5}, x_{6}, x_{8}\right\}\right\}=\operatorname{co}\left\{\zeta_{a}\left\{x_{1}, x_{4}, x_{7}\right\}\right\} \\
=\operatorname{co}\left\{x_{1}, x_{4}, x_{7}\right\}=\operatorname{co}\left\{x_{2}, x_{3}, x_{5}, x_{6}, x_{8}\right\}\end{array}$ \\
\hline Degree of Accuracy & $\frac{\left|x_{2}, x_{3}, x_{5}, x_{6}, x_{8}\right|}{\left|x_{2}, x_{3}, x_{5}, x_{6}, x_{8}\right|}=\frac{5}{5}=1$ \\
\hline
\end{tabular}

\subsubsection{Constructing a pretopology when removing the attribute Reference}

Table 12: shows the pretopology obtained when Table 13: shows the normalization to make the numbers removing the attribute reference. in the interval $[0,1]$ obtained from the information system.

\begin{tabular}{|l|c|c|c|c|c|c|c|c|}
\hline & $x_{1}$ & $x_{2}$ & $x_{3}$ & $x_{4}$ & $x_{5}$ & $x_{6}$ & $x_{7}$ & $x_{8}$ \\
\hline$x_{1}$ & 0 & $\frac{5}{6}$ & $\frac{5}{3}$ & $\frac{3}{2}$ & $\frac{2}{3}$ & $\frac{3}{2}$ & $\frac{3}{2}$ & $\frac{7}{3}$ \\
\hline$x_{2}$ & $\frac{5}{6}$ & 0 & $\frac{5}{6}$ & $\frac{4}{3}$ & $\frac{3}{2}$ & $\frac{4}{3}$ & $\frac{3}{2}$ & $\frac{3}{2}$ \\
\hline$x_{3}$ & $\frac{5}{3}$ & $\frac{5}{6}$ & 0 & $\frac{3}{2}$ & $\frac{5}{3}$ & $\frac{3}{2}$ & $\frac{13}{6}$ & $\frac{2}{3}$ \\
\hline$x_{4}$ & $\frac{3}{2}$ & $\frac{4}{3}$ & $\frac{3}{2}$ & 0 & $\frac{5}{6}$ & 0 & $\frac{4}{3}$ & $\frac{13}{6}$ \\
\hline$x_{5}$ & $\frac{2}{3}$ & $\frac{3}{2}$ & $\frac{5}{3}$ & $\frac{5}{6}$ & 0 & $\frac{5}{6}$ & $\frac{13}{6}$ & $\frac{7}{3}$ \\
\hline$x_{6}$ & $\frac{3}{2}$ & $\frac{4}{3}$ & $\frac{3}{2}$ & 0 & $\frac{5}{6}$ & 0 & $\frac{4}{3}$ & $\frac{13}{6}$ \\
\hline$x_{7}$ & $\frac{3}{2}$ & $\frac{3}{2}$ & $\frac{13}{6}$ & $\frac{4}{3}$ & $\frac{13}{6}$ & $\frac{4}{3}$ & 0 & $\frac{3}{2}$ \\
\hline$x_{8}$ & $\frac{7}{3}$ & $\frac{3}{2}$ & $\frac{2}{3}$ & $\frac{13}{6}$ & $\frac{7}{3}$ & $\frac{13}{6}$ & $\frac{3}{2}$ & 0 \\
\hline
\end{tabular}

\begin{tabular}{|l|c|c|c|c|c|c|c|c|}
\hline & $\boldsymbol{x}_{1}$ & $\boldsymbol{x}_{2}$ & $\boldsymbol{x}_{3}$ & $\boldsymbol{x}_{4}$ & $\boldsymbol{x}_{5}$ & $\boldsymbol{x}_{6}$ & $\boldsymbol{x}_{7}$ & $\boldsymbol{x}_{8}$ \\
\hline$x_{1}$ & 0 & 0.5 & 0.7 & 0.6 & 0.2 & 0.6 & 0.6 & 1 \\
\hline $\boldsymbol{x}_{2}$ & 0.3 & 0 & 0.3 & 0.6 & 0.6 & 0.6 & 0.6 & 0.6 \\
\hline $\boldsymbol{x}_{3}$ & 0.7 & 0.5 & 0 & 0.6 & 0.7 & 0.6 & 1 & 0.2 \\
\hline$x_{4}$ & 0.6 & 0.8 & 0.6 & 0 & 0.3 & 0 & 0.6 & 0.9 \\
\hline$x_{5}$ & 0.2 & 1 & 0.7 & 0.3 & 0 & 0.3 & 1 & 1 \\
\hline$x_{6}$ & 0.6 & 0.8 & 0.6 & 0 & 0.3 & 0 & 0.6 & 0.9 \\
\hline$x_{7}$ & 0.6 & 1 & 1 & 0.6 & 0.9 & 0.6 & 0 & 0.6 \\
\hline$x_{8}$ & 1 & 1 & 0.3 & 1 & 1 & 1 & 0.6 & 0 \\
\hline
\end{tabular}

Therefore, the pretopology component of matrix by using Eq. (1)

$$
\begin{aligned}
& \zeta_{a_{5}}\left\{x_{1}\right\}=\left\{x_{1}, x_{2}\right\} \\
& \zeta_{a_{5}}\left\{x_{2}\right\}=\left\{x_{2}\right\} \\
& \zeta_{a_{5}}\left\{x_{3}\right\}=\left\{x_{2}, x_{3}\right\} \\
& \zeta_{a_{5}}\left\{x_{4}\right\}=\left\{x_{4}\right\} \\
& \zeta_{a_{5}}\left\{x_{5}\right\}=\left\{x_{5}\right\} \\
& \zeta_{a_{5}}\left\{x_{6}\right\}=\left\{x_{6}\right\} \\
& \zeta_{a_{5}}\left\{x_{7}\right\}=\left\{x_{7}\right\} \\
& \zeta_{a_{5}}\left\{x_{8}\right\}=\left\{x_{8}\right\}
\end{aligned}
$$

\section{Therefore,}


1- Calculate $\zeta_{a}\{\text { Decision }\}_{\text {Accept }}$ and $\operatorname{int}_{\zeta_{a}}\{\text { Decision }\}_{\text {Accept }}$

\begin{tabular}{|c|c|}
\hline$\zeta_{a}\{\text { Decision }\}_{\text {Accept }}$ & $\zeta_{a}\left\{x_{1}, x_{4}, x_{7}\right\}=\left\{x_{1}, x_{2}, x_{4}, x_{7}\right\}$ \\
\hline int $_{\zeta_{a}}\{\text { Decision }\}_{\text {Accept }}$ & $\begin{aligned} \operatorname{int}_{\zeta_{a}}\left\{x_{1}, x_{4}, x_{7}\right\} & =\operatorname{co}\left\{\zeta_{a} \operatorname{co}\left\{x_{1}, x_{4}, x_{7}\right\}\right\}=\operatorname{co}\left\{\zeta_{a}\left\{x_{2}, x_{3}, x_{5}, x_{6}, x_{8}\right\}\right\} \\
= & \operatorname{co}\left\{x_{2}, x_{3}, x_{5}, x_{6}, x_{8}\right\}=\left\{x_{1}, x_{4}, x_{7}\right\}\end{aligned}$ \\
\hline Degree of Accuracy & $\frac{\left|x_{1}, x_{4}, x_{7}\right|}{\left|x_{1}, x_{2}, x_{4}, x_{7}\right|}=\frac{3}{4}=0.75$ \\
\hline
\end{tabular}

2- Calculate $\zeta_{a}\{\text { Decision }\}_{\text {Reject }}$ and int $_{\zeta_{a}}\{\text { Decision }\}_{\text {Reject }}$

\begin{tabular}{|l|c|}
\hline \multicolumn{1}{|c|}{$\zeta_{a}\{\text { Decision }\}_{\text {Reject }}$} & $\zeta_{a}\left\{x_{2}, x_{3}, x_{5}, x_{6}, x_{8}\right\}=\left\{x_{2}, x_{3}, x_{5}, x_{6}, x_{8}\right\}$ \\
\hline int $_{\zeta_{a}}\{\text { Decision }\}_{\text {Reject }}$ & int $_{\zeta_{a}}\left\{x_{2}, x_{3}, x_{5}, x_{6}, x_{8}\right\}=\operatorname{co}\left\{\zeta_{a}\left\{x_{2}, x_{3}, x_{5}, x_{6}, x_{8}\right\}\right\}=\operatorname{co}\left\{\zeta_{a}\left\{x_{1}, x_{4}, x_{7}\right\}\right\}$ \\
& $=\operatorname{co}\left\{x_{1}, x_{2}, x_{4}, x_{7}\right\}=\operatorname{co}\left\{x_{3}, x_{5}, x_{6}, x_{8}\right\}$
\end{tabular}

\subsection{Resulting Reduction from Information System}

Table 14: shows the degree of accuracy in all previous tables:

\begin{tabular}{|c|c|c|c|c|c|}
\hline $\begin{array}{c}\text { Degree of } \\
\text { Accuracy }\end{array}$ & $\begin{array}{c}\text { Original } \\
\text { Pretopology }\end{array}$ & $\begin{array}{c}\text { After removing } \\
\text { Diploma }\end{array}$ & $\begin{array}{c}\text { After removing } \\
\text { French }\end{array}$ & $\begin{array}{c}\text { After removing } \\
\text { Experience }\end{array}$ & $\begin{array}{c}\text { After removing } \\
\text { Reference }\end{array}$ \\
\hline$\{\text { Decision }\}_{\text {Accept }}$ & 0.5 & 0.5 & 0.25 & 1 & 0.75 \\
\hline$\{\text { Decision }\}_{\text {Reject }}$ & 0.6 & 0.6 & 0.57 & 1 & 0.8 \\
\hline
\end{tabular}

From the above table, when using the method of reduction attributes mentioned in (3.4), we can conclude that:

The core $=\{$ Diploma, French $\}$.

\section{CONCLUSION}

In this paper, several pretopological spaces were constructed from some information system, as well as the definitions of some essential operations for the pretopological space and a brief study of its properties were presented. A condition was set in order to be able to create a matrix that represents the information system's pretopology, considering that this condition should verify the two axioms of the pretopological space's definition. For validation, an example was given to connect the structure of a pretopological space. Moreover, we proposed a methodology for attributes reduction by measuring the degree of accuracy of information system attributes.

\section{REFERENCE}

[1] Al-shami, T. M., Information $\quad$ Sciences, $569 \quad 110-124, \quad 2021$, https://doi.org/10.1016/j.ins.2021.04.016.

[2] Al-shami, T. M., Soft Computing, 2021, https://doi.org/10.1007/s00500-021-06358-0.

[3] Al-shami, T. M., Alshammari, I., and El-Shafei, M.E., Journal of Intelligent and Fuzzy Systems, 41(1), 1393-1406, 2021, doi: 10.3233/JIFS-210272.

[4] Al-shami, T. M., Fu, W. Q. and Abo-Tabl, E. A., Complexity, Volume 2021, Article ID 6666853, 6 pages, 2021, https://doi.org/10.1155/2021/6666853. 
[5] Auray, J.-P., Duru, G., and Mougeot, M., Economics letter, 2(4), 1979, https://doi.org/10.1016/0165-1765(79)90048-X.

[6] Belmandt, Z., Hermann, 2011, doi: https://hal.archives-ouvertes.fr/hal-00605389 .

[7] Belmandt, Z., Manuel de prétopologie et ses applications. Hermès, 1993.

[8] Brissaud, M., Les espaces prétopologiques. Compte-rendu de l'Académie des Sciences, 280(A):705708, 1975.

[9] Dalud-Vincent, M., Brissaud, M., and Lamure, M., International Journal of Pure and Applied Mathematics, pages 391-402, Jan. 2009, doi: https://halshs.archives-ouvertes.fr/halshs-01778084.

[10] El-Bably, M. K., and Al-shami, T. M., International Journal of Biomathematics, 2021, https://dx.doi.org/10.1142/S1793524521500868.

[11] Gil-Aluja, J., and Lafuente, A. M. G., economic phenomena, springer 2012, doi: 10.1007/978-3642-24812-2.

[12] Largeron, C., and Bonnevay, S., Information Sciences, 144(1-4):169 - 185, 2002, doi:10.1016/S0020-0255(02)00189-5.

[13] Levorato, V., and Bui, M., In IEEE, editor, (I2CS), pages 122-134, Fort de France, Martinique, June 2008, doi: https://tel.archives-ouvertes.fr/tel-02147578.

[14] Kuratowski, K., Topologie. Nakł. Polskiego Towarzystwa Matematycznego, Warszawa, 1952. OCLC: 3014396.

[15] Nasef, A. A., Jafari, S., Caldas, M., Latif, R. M., and Azzam, A. A., journal of linear topological algebra, $\quad 7(1), \quad 1-9, \quad 2018, \quad$ doi: http://jlta.iauctb.ac.ir/article_539112_84a8805135ae21260c5ffc3d8ce51df2.

[16] Pawlak, Z., Dordrecht, Boston, London: Kluwer Academic Publishers; 1991, doi: 10.1007/978-94011-3534-4.

[17] Zhexue Huang,

http://citeseerx.ist.psu.edu/viewdoc/download?doi=10.1.1.134.83\&rep=rep1\&type=pdf 